pitalisation. Phenylacetate hydrolase activity was assayed on these blood samples using serum vacutainer tubes to avoid calcium chelation. The colorimetric assay was run as described (5) using phenylacetate as substrate. Phenylacetate degrading activity of PON1 is independent of the coding region polymorphisms (position 55 and 192) of this gene and thus is more reflective of the concentration of PON1 protein present (7). It is unclear whether this activity reflects the actual activity of the PON1 towards endogenous substrates such as HTL. Neither HTL degrading activity, nor HTL concentrations have been assayed in this study. The precise HTL level in human plasma has been reported for six persons (8).

An inverse correlation was found between Hcy concentration and phenylacetate hydrolase activity $(\mathrm{r}=0.36, \mathrm{y}=-0.34 \mathrm{x}+$ $22,01, \mathrm{ddl}=110, \mathrm{t}=4,09, \mathrm{p}<0.001-$ Fig. 1). Thus, the level of Hcy appears to be one of the determinants of phenyl-acetate hydrolase activity in serum. While several mechanisms could account for such an effect, the observations made in hyperhomocysteinemic mice suggest that Hcy could negatively regulate PON1 gene expression in the liver which would result in decreased serum PON1 activity. It is unknown whether this Hcy effect is direct or not, however, the fact that Hcy is itself a product of PON1 activity suggests an autoregulatory mechanism whereby increased Hcy concentrations lead to the retroinhibition of the enzyme gene expression.
The observations shown here could have implications for the delineation of the mechanism of Hcy action. Indeed, it is known that altered PON1 activity as observed in individuals carrying the polymorphisms at position 55 and 192 constitutes a susceptibility marker for cardiovascular disease. This is likely due to the lipoproteins-protective anti oxidant effect of PON1 and possibly to its ability to degrade HTL. The repression of serum PON1 activity could be one of the steps leading to Hcy toxicity. Alternatively, our data also suggest that PON1 level could be an additional determinant of the Hcy plasma concentration.

\section{Acknowledgments}

This work was supported by the European Union Grant (QLRT-2001-00816) and a grant "vin et santé". I thank Anna Kostrzack for their technical assistance

\section{Nathalie Janel ${ }^{2}$, Karine Robert ${ }^{2}$, Karine Demuth ${ }^{3}$, Cédric Gouédard', Robert Barouki', 3, Jean François Chassél, 3 IINSERM UMR S-490, Université Paris V, Paris, France \\ ${ }^{2}$ EA 3508, Université Paris 7, Paris, France}

${ }^{3}$ Service de Biochimie, Hopital Europeen Georges Pompidou, Paris, France

\section{References}

1. Glowacki R, Jakubowski H Cross-talk between CYS-34 and lysine residues in human serum albumin revealed by N-homocysteinylation. J Biol Chem 2004; 279: 10864-71.

2. Jakubowski H, Ambrosius WT, Pratt JH. Genetic determinants of homocysteine thiolactonase activity in humans: implications for atherosclerosis. FEBS Lett 2001; 491: 35-9.

3. Voetsch B, Loscalzo. Genetic determinants of the arterial thrombosis. Arterioscler Thromb Vasc Biol 2004; 24: 216-29.
4. Jakubowski H. Calcium-dependant human serum homocysteine thiolactone hydrolase. J Biol Chem 2000; 275: 3957-62.

5. Robert K, Chassé JF, Santiard-Baron D et al. Altered gene expression in liver from a murine model of hyperhomocysteinemia. J Biol Chem 2003; 278 . 31504-11.

6. Janel N, Robert K, Chabert $\mathrm{C}$ at al. Mouse liver paraoxonase-1 gene expression is downregulated in hyperhomocysteinemia. Thromb Haemost 2004; 92 221-2.
7. Jarvik GP, Hatsukami TS, Carlson C et al. Paraoxonase activity, but not haplotype utilizing the linkage disequilibrium structure, predicts vascular disease. Arterioscler Thromb Vasc Biol 2003; 23: 1465-71.

8. Jakubowski H. The determination of homocysteine-thiolactone in biological samples. Anal Biochem 2002; 308: 112-9.

\title{
Elastic stockings, hydroxyethylrutosides or both for the treatment of post-thrombotic syndrome
}

\section{Dear Sir,}

At least one of every three patients with deep-vein thrombosis (DVT) of the lower extremities will develop post-thrombotic sequelae within 5 years $(1,2)$. The established post-thrombotic

\footnotetext{
Correspondence to:

Paolo Prandoni

Department of Medical and Surgical Sciences

$2^{\text {nd }}$ Chair of Internal Medicine

University of Padua, Italy

Tel.: +39-049-8212656, Fax: +39-049-821266|

E-mail: paoloprandoni@tin.it

Received August 15, 2004

Accepted after October 19, 2004
}

Thromb Haemost 2005; 93: I 83-5 syndrome (PTS) remains a significant cause of chronic ill health, with considerable socio-economic consequences for both the patient and the health care services (3).

In contrast with the extensive documentation available on epidemiology, diagnosis, and prevention of PTS, little is known about the treatment of this common complication of DVT (4). Once the PTS is established, treatment options are aimed at limiting progression to an ulcer with the use of graduated elastic compression stockings (ECS), which reduce venous hypertension and improve microcirculation (5). Besides compression therapy, rutosides are commonly used for prevention and treatment of several manifestations of chronic venous insufficiency (6). In a prospective, controlled, randomized pilot study, we compared 
the efficacy of ECS, hydroxyethylrutosides (HR), or both for the treatment of established PTS in a consecutive series of patients who had experienced at least one episode of DVT.

All consecutive patients with clinical symptoms and signs suggestive of PTS referring to our institution were potentially eligible for the study, provided that they had a history of ultrasound proven proximal-vein thrombosis of the qualifying leg. Patients who had skin ulcers were excluded from the study, as were patients who were already wearing compression stockings, patients with a short life expectancy, and those who had a contraindication for the use of either stockings (e.g. advanced-stage peripheral arterial insufficiency) or rutosides (e.g., peptic ulcer). The presence and severity of post-thrombotic signs and symptoms was assessed by a single trained investigator using a standardised and validated score (the Villalta scale) $(1,17)$. Each of five symptoms (pain, cramps, heaviness, pruritus, and paresthesia) and six signs (pretibial oedema, induration of the skin, hyperpigmentation, new venous ectasia, redness, and pain during calf compression) received a score ranging from 0 to 3 using the contralateral unaffected leg as denominator. PTS was defined as severe in case of a score higher than 14 , and mild in case of a score between 5 and 14. The study was approved by the Institutional Review Board.

At the time of examination, consenting patients were randomly allocated to wear a below-knee graded ECS ( 30 to $40 \mathrm{~mm}$ $\mathrm{Hg}$ at the ankle) of the affected leg, to receive oral treatment with HR (1000 mg twice a day in a soluble powder), or both, for a period of one year according to a computer-generated list. All patients were seen at three and six months after randomisation, and then after completion of 12 months. Each assessment was carried out by an investigator who was aware of the side of the index venous thrombosis, but was unaware of the treatment allocation and of the results of previous measurements. Patients were considered as having experienced a PTS improvement when the PTS score became lower than 5 and/or decreased by at least $30 \%$ in comparison to the baseline one at any time during the follow-up. Patients whose score increased by at least $30 \%$ were considered as having undergone a PTS worsening. Compliance to stockings was defined as satisfactory if stockings were reportedly used for at least $80 \%$ during daytime. Similarly, compliance to HR was defined as satisfactory if at least $80 \%$ of the study drug had reportedly been taken.

Statistical analysis was done on an intention-to-treat basis. Hazard ratios and their $95 \%$ confidence interval (CI) for PTS improvement and worsening of ECS versus HR, ECS versus the combination, and HR versus the combination were calculated using Cox regression models. Hazard ratios were adjusted for age, sex, body weight, modality of clinical presentation (i.e., spontaneous or associated with recognisable risk factors), location of the thrombotic episode, history of multiple ipsilateral thrombotic episodes, time elapsed between DVT episode and patient's recruitment, and PTS severity. SPSS, version 10, Chicago, was the statistical package used for the analysis.

Out of 156 eligible patients, 120 were recruited for this investigation, 40 in each study group. Table 1 shows the main baseline characteristics of the enrolled patients. There were more males in the ECS group, while in the ECS + HR group there was a higher rate of thrombosis involving both the popliteal and the femoral
Table I: Main characteristics of study patients at presentation.

\begin{tabular}{|c|c|c|c|}
\hline & $\begin{array}{l}\text { ECS } \\
(N=40)\end{array}$ & $\begin{array}{l}H R \\
(N=40)\end{array}$ & $\begin{array}{l}\text { ECS + HR } \\
(\mathrm{N}=40)\end{array}$ \\
\hline Age (median, range) & 73 (24-9I) & $68.5(32-87)$ & $66.5(33-89)$ \\
\hline Males & 17 & 9 & 10 \\
\hline $\begin{array}{l}\text { Months since DVT } \\
\text { (median, range) }\end{array}$ & $21(6-120)$ & $18(6-120)$ & $14(6-120)$ \\
\hline Risk factors for DVT & $20(50.0 \%)$ & $21(52.5 \%)$ & $24(60.0 \%)$ \\
\hline - obesity & 2 & 8 & 5 \\
\hline - cancer & 7 & 8 & 7 \\
\hline - trauma & 3 & 3 & 3 \\
\hline - surgery & 3 & 2 & 8 \\
\hline - immobilization & I & I & I \\
\hline - estrogens & I & 0 & 0 \\
\hline - pregnancy & 0 & 1 & 0 \\
\hline - thrombophilia & 5 & I & 6 \\
\hline - previous DVT & 4 & 2 & 4 \\
\hline \multicolumn{4}{|l|}{ Location of DVT } \\
\hline - popliteal only & 25 & 30 & 20 \\
\hline - femoral only & 3 & 2 & 2 \\
\hline - popliteal and femoral & 12 & 8 & 18 \\
\hline \multicolumn{4}{|l|}{ Severity of PTS } \\
\hline - mild to moderate & 37 & 35 & 39 \\
\hline - serious & 3 & 5 & 1 \\
\hline
\end{tabular}

vein and a lower rate of severe PTS. Three patients per group were lost to follow-up, on average after six months. During the study period 2 patients died, both belonging to the ECS group, and 1 patient per group experienced a recurrent thromboembolism.

Of the 80 patients allocated to ECS, alone or combined with HR, only 2 patients $(2.5 \%)$ could not tolerate them. Of the remaining 78 patients, 70 wore their stockings for at least $80 \%$ of daytime during the study period. Of the 80 patients allocated to HR, alone or associated with ECS, 6 patients (7.5\%) stopped using it after a short period of time, mostly because of gastric pain. Of the remaining 74 patients, 64 took at least $80 \%$ of the study medication during the whole observation period.

During the study period, a PTS improvement was observed in 28 patients receiving ECS, 26 treated with HR, and 25 allocated to the combination; a PTS worsening in 6,9, and 9, respectively. The adjusted hazard ratios for PTS improvement for ECS versus HR, ECS alone versus the combination, and HR alone versus the combination were 1.18 (0.67 to 2.09), 1.29 (0.73 to 2.29), and 0.92 ( 0.50 to 1.68), respectively; and hazard ratios for PTS worsening 0.80 ( 0.27 to 2.37$), 0.73$ ( 0.25 to 2.19 ), and 1.22 ( 0.47 to 3.23 ), respectively.

A few methodological aspects require comment. First, because of a relatively small sample size, and the correspondingly wide $95 \%$ confidence intervals around the observed hazard ratios, the results of this study have to be interpreted with caution. 
Second, the true efficacy of the investigated strategies could not be properly assessed because of the lack of a control group without treatment. Finally, although the combination of mechanical and therapeutic regimens did not show superiority over ECS or HR, when administered alone, it cannot be excluded.

The results of this pilot randomised study suggest that elastic compression stockings and flavonoid rutosides are equally effective for improving the clinical manifestations of PTS arising in patients with previous DVT. Larger studies are needed to confirm these conclusions.

Michela Frulla, Antonio Marchiori, Donatella Sartor, Laura Mosena, Daniela Tormene, Alessia Concolato, Larissa Hartman, Paolo Prandoni

Department of Medical and Surgical Sciences, $2^{\text {nd }}$ Chair of Internal Medicine, University of Padua, Italy

\section{References}

1. Prandoni P, Lensing AWA, Cogo A, et al. The longterm clinical course of acute deep venous thrombosis. Ann Intern Med 1996; 125: 1-7.

2. Mohr DN, Silverstein MD, Heit JA, et al. The venous stasis syndrome after deep venous thrombosis or pulmonary embolism: a population-based study. Mayo Clin Proc 2000; 75: 1249-56.

3. Bergqvist D, Jendteg S, Johansen L, et al. Cost of long-term complications of the lower extremities: an analysis of a defined patient population in Sweden Ann Intern Med 1997; 126: 454-7.

4. Kahn SR, Ginsberg JS. Relationship between deep venous thrombosis and the postthrombotic syndrome Arch Intern Med 2004; 164: 17-26.

5. Kahn SR, Elman E, Rodger MA, et al. Use of elastic compression stockings after deep venous thrombosis: a comparison of practices and perceptions of thrombosis physicians and patients. J Thomb Haemost 2003; 1: 500-6.
6. Neumann HAM. Medical treatment for venous diseases: the role of rutosides in daily practice. Phlebology Digest 2003; 16: 5-10.

7. Kahn SR, Hirsch A, Shrier I. Effect of post-thrombotic syndrome on health-related quality of life after deep venous thrombosis. Arch Intern Med 2002; 162 : $1144-8$ 